Portland State University

PDXScholar

1989

\title{
Endless state-of-polarization control for coherent optical communication systems using nematic liquid crystals
}

Scott Hamilton Rumbaugh

Portland State University

Follow this and additional works at: https://pdxscholar.library.pdx.edu/open_access_etds

Part of the Electrical and Computer Engineering Commons Let us know how access to this document benefits you.

\section{Recommended Citation}

Rumbaugh, Scott Hamilton, "Endless state-of-polarization control for coherent optical communication systems using nematic liquid crystals" (1989). Dissertations and Theses. Paper 4145.

https://doi.org/10.15760/etd.6029

This Thesis is brought to you for free and open access. It has been accepted for inclusion in Dissertations and Theses by an authorized administrator of PDXScholar. Please contact us if we can make this document more accessible: pdxscholar@pdx.edu. 
AN ABSTRACT OF THE THESIS OF Scott Hamilton Rumbaugh for the Master of Science in Electrical Engineering presented December 7, 1989.

Title: Endless State-of-Polarization Control for Coherent Optical Communication Systems using Nematic Liquid Crystals

APPROVED BY THE MEMBERS OF THE THESIS COMMITTEE

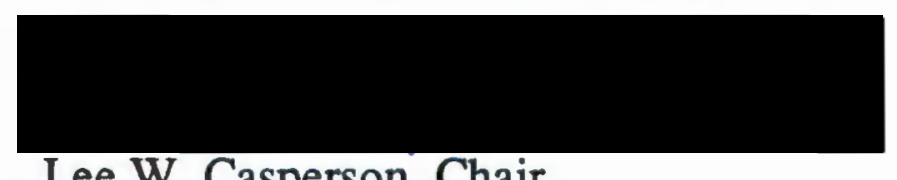

Lee W. Casperson, Chair

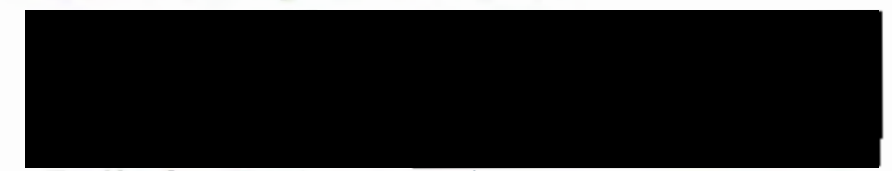

Rajinder P. Aggarwal

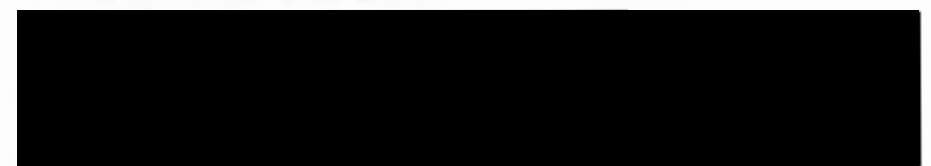

David Paul

One of the obstacles to coherent fiber-optic communications is the unpredictable polarization drift which necessitates the use of an active polarization controlling system to match the polarizations of the signal and local oscillator. The polarization match must be maintained during the reset of any of the finite range components to prevent loss of data. We present a novel and practical system which uses three liquid crystal devices for the polarization matching process. Also, the required reset control algorithm and its derivation are described in detail. 


\title{
ENDLESS STATE-OF-POLARIZATION CONTROL FOR COHERENT OPTICAL COMMUNICATION SYSTEMS USING NEMATIC LIQUID CRYSTALS
}

\author{
by \\ SCOTT H. RUMBAUGH
}

A thesis submitted in partial fulfillment of the requirements for the degree of

\author{
MASTER OF SCIENCE \\ in \\ ELECTRICAL ENGINEERING
}

Portland State University

1990 
TO THE OFFICE OF GRADUATE STUDIES:

The members of the commitee approve the thesis of Scott Hamilton Rumbaugh presented December 7, 1989.

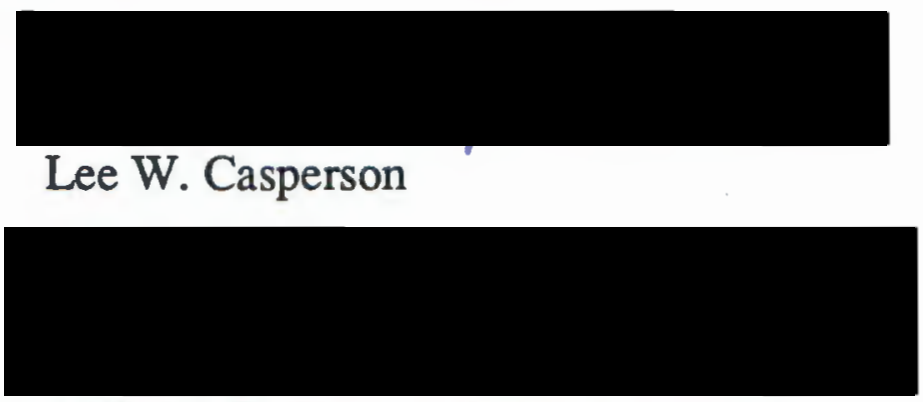

$$
\text { Rajinder P. Aggarwal }
$$

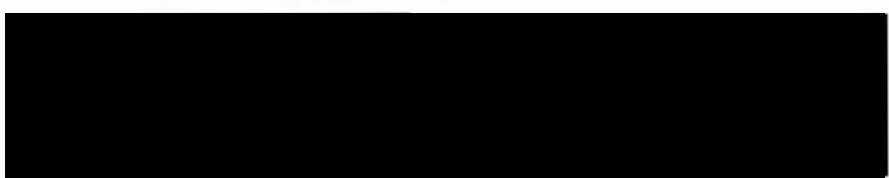

David Paul

\section{APPROVED:}

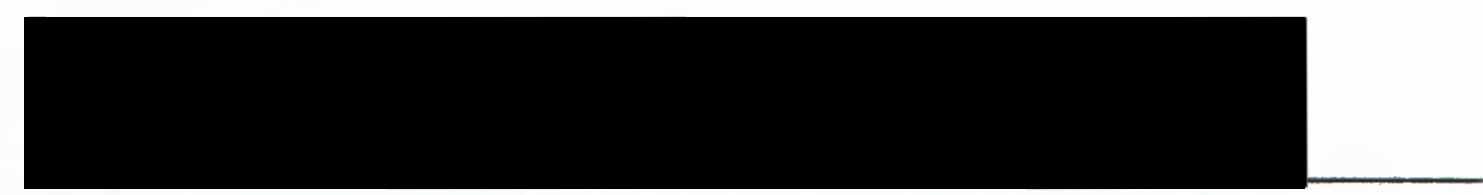

Rolf Schauman, Chair, Department of Electrical Engineering

C. William Savery, Interim Vice $\bar{P}$ rovost for Graduate Studies and Research 


\section{ACKNOWLEDGEMENTS}

I am very grateful to Professor Lee Casperson for his guidance and inspiration during my research at Portland State University. I would also like to thank Michael Jones for his valuable contributions, Phil Bos and Rickey Koehler-Beran for many helpful discussions and for providing the liquid crystal devices, and Brent Jensen for designing and building the electronic circuitry used in the experiments. 


\section{TABLE OF CONTENTS}

\section{PAGE}

ACKNOWLEDGEMENTS iii

LIST OF FIGURES

\section{CHAPTER}

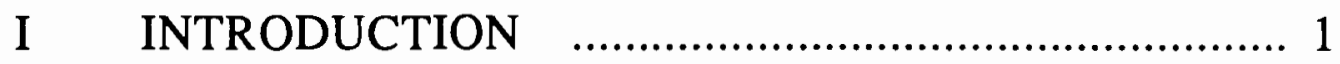

II COHERENT DETECTION ..................................... 3

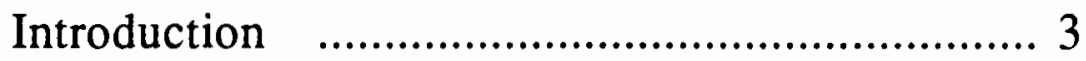

The Importance of a Polarization Match ............... 4

III LIQUID CRYSTAL RETARDERS …....................... 8

Introduction

Liquid Crystal Retarders ….............................. 8

IV SYSTEM OPERATION ........................................ 13

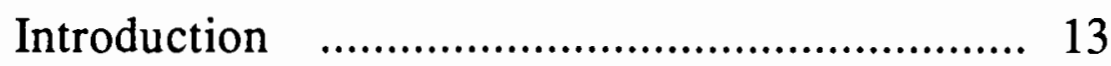

Control Algorithm ........................................ 14

Normal Mode of Operation …........................... 14

Reset Mode of Operation …................................. 16

Derivation of Upper Range Reset

Derivation of Lower Range Reset

V EXPERIMENTAL VERIFICATION ........................ 28

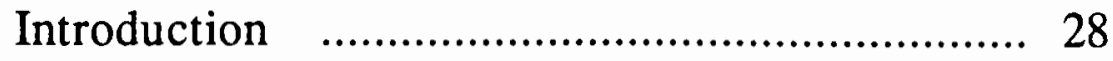


Experimental Apparatus ................................ 28

Characterization of Retarders ............................ 29

Rotator Analog …........................................ 30

System Demonstration ….................................. 31

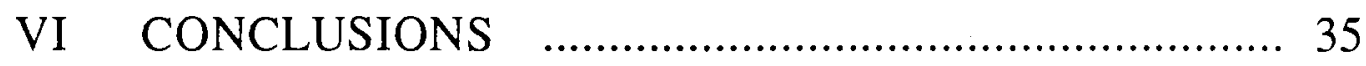

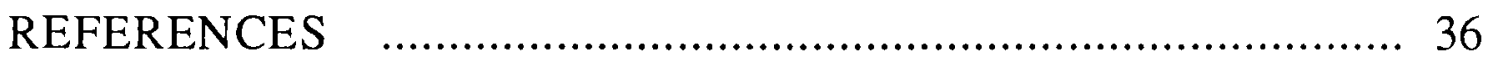

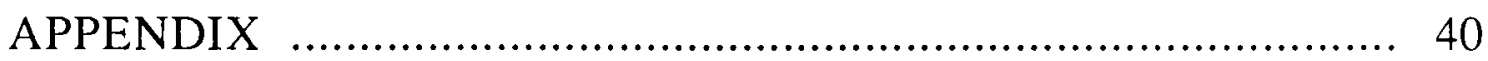




\section{LIST OF FIGURES}

FIGURE

PAGE

1. Implementation of an SOP matching system in

fiber-optic heterodyne detection ........................... 7

2. Nematic liquid crystal retarder with $\mathrm{V}<\mathrm{Vth}$................ 11

3. Nematic liquid crystal retarder with $\mathrm{Vth}<\mathrm{V}<\mathrm{V} \max \ldots . .11$

4. Nematic liquid crystal retarder with $V=V \max \quad \ldots \ldots \ldots \ldots . . .11$

5. The retardance as a function of applied voltage for a

typical liquid crystal device ............................... 12

6. The SOP matching system showing the orientation

and retardance of the liquid crystal devices …......... 27

7. A Poincare sphere representation of SOP matching

during the normal mode of operation .................... 27

8. Experimental apparatus ........................................... 32

9. A plot of $V_{d 1}$ and $V_{d 2}$ for several tilt angles of the

polarization .................................................... 32

10. A plot of the polarization states used in demonstrating

endless and complete polarization control

11. A plot of the control voltages demonstrating normal

and reset modes

12. The Poincare sphere ................................................. 43

13. An example using the Poincare sphere .......................... 43 


\section{CHAPTER I}

\section{INTRODUCTION}

Modern fiber-optic communications systems employ direct detection of intensity modulated signals. Coherent communication offers the potential of increasing channel capacity by a factor of 1000 and receiver sensitivity by 14-20 dB [1], which would also lead to a significant increase in repeater spacing [2]-[4]. Coherent communication has come closer to realization with the advent of narrow spectral linewidth semiconductor lasers and single-mode fibers [5]. However, signal polarization fluctuations, due to inherent birefringent properties of the fiber and those induced by thermal and mechanical stresses, present an obstacle to heterodyne detection since coherent detection requires that the polarization of the local oscillator (LO) and signal match. Nevertheless, the polarization of signals in installed fibers varies slowly enough to permit SOP compensation [6], and state of polarization (SOP) matching schemes may be implemented in existing fiber-optic networks.

The distinct advantages of coherent communications are not limited to long-haul telecommunications. The capabilities of network environments can also be significantly increased [7]. Coherent detection would allow for optical frequency division multiplexing (OFDM), in place of conventional wavelength division multiplexing (WDM), which would increase channel throughput and capabilities of the network. The increased receiver sensitivity would also permit the implementation of a passive star coupler to link users so that conventional star or point to point links would 
no longer be needed. Also, since the hub of this network is passive, no optic-electronic and electronic-optic conversions are necessary.

A practical SOP controlling system must change the polarization endlessly to prevent momentary signal loss during the reset of any constituent components. Currently, various groups are testing systems which use mechanically induced birefringence as a means of matching the signal SOP to that of the LO [8]-[14]. Most other research has concentrated on the use of $\mathrm{LiNiBO}_{3}$ waveguides [15]-[18], while other proposed systems utilize electrooptic crystals, Faraday rotators, or rotating wave-plates [19], [20]. Difficulties associated with these systems include mechanical fatigue, high operating voltages, and excessive cost. To date, no practical systems have been reported.

We have developed an endless SOP controlling system for use in coherent fiber-optic detection systems which does not exhibit the difficulties associated with previous systems [21], [22]. It uses three polarization controlling devices constructed with nematic liquid crystals. These cells are inexpensive to construct, have no moving parts, and can be operated on a $10-30 \mathrm{~V}$ supply.

This thesis describes the operation of liquid crystal retarders and their application in a polarization controlling system. A description of optical heterodyne detection and the effects of mis-matched polarization states is given in Chapter II. Chapter III provides a brief description of liquid crystals and their use in polarization controlling devices. Chapter IV is an in-depth description of the system control and includes the derivation of the control algorithm. The system is experimentally demonstrated in Chapter V. 


\section{CHAPTER II}

\section{COHERENT DETECTION}

\section{INTRODUCTION}

A heterodyne system which implememts SOP matching is illustrated in Figure 1. The signal beam with a carrier frequency $\omega$ is spatially combined on the detector with a local oscillator offset by a frequency $\delta \omega$. Photo-detectors are inherently square law devices so that an interference (IF) signal is generated at the offset frequency $\delta \omega$. This interference signal is proportional to the strength of the $\mathrm{LO}$ and depends on how closely the polarizations of the LO and the signal match. The SOP controlling system continuously compensates the LO SOP to match that of the signal to prevent signal fading. This is accomplished by an SOP controlling system in which the only feedback available is the interference signal strength. In order to prevent momentary signal loss the control needs to be complete in that all possible polarization states can be matched, and endless so that no change in the SOP occurs during the reset of any of the finite range polarization controlling components.

In order to understand the importance of polarization matching in coherent detection systems, we will examine the interference signal in two orthogonal decompostions of the polarizations of both the signal and LO beams. Since any polarization may be described as a superposition of linear polarizations in orthogonal directions, we will describe each beam 
in terms of linear polarizations along the $\mathrm{x}$ and $\mathrm{y}$ directions transverse to the direction of propagation. The total IF signal is the sum of the IF signals of the fields in each direction and is maximum when the individual signals are in phase and of maximum amplitude. It will be shown below that relative phase and magnitudes of these IF signals are determined by how closely the polarizations are matched.

\section{THE IMPORTANCE OF A POLARIZATION MATCH}

The total electric field of a coherent beam is described as a superposition of linearly polarized beams below

$$
\mathbf{E}=\mathrm{E}_{\mathrm{x}} \cos \left(\omega \mathrm{t}+\varepsilon_{\mathrm{x}}\right) \widehat{\mathbf{x}}+\mathrm{E}_{\mathrm{y}} \cos \left(\omega \mathrm{t}+\varepsilon_{\mathrm{y}}\right) \widehat{\mathbf{y}}
$$

where $\omega$ is the carrier frequency, $E_{x}$ and $E_{y}$ are the magnitudes of the electric field in the $\mathrm{x}$ and $\mathrm{y}$ directions and $\varepsilon_{\mathrm{x}}$ and $\varepsilon_{\mathrm{y}}$ are relative phase terms of the components. The polarization is determined by the ratio of the magnitudes and the relative phase of the components. The intensity of the field in $(2-1)$ is $\left(\mathrm{E}_{\mathbf{x}}{ }^{2}+\mathrm{E}_{\mathbf{y}}{ }^{2}\right)^{1 / 2}$.

The signal beam may be intensity or angle (phase or frequency) modulated and in the most general form will be given by

$$
E_{s}=E_{s x}(t) \cos \left(\omega t+\theta(t)+\varepsilon_{x}\right) \widehat{x}+E_{s y}(t) \cos \left(\omega t+\theta(t)+\varepsilon_{y}\right) \widehat{y}
$$

In angle modulation, $\theta(t)$ carries the signal information, but the total phase difference between the $\mathrm{x}$ and $\mathrm{y}$ components will remain constant, for constant polarization. For an amplitude modulated signal, $\theta(t)$ will be 0 
and the magnitude of the quadrature components is modulated with $E_{s x}(t) / E_{s y}(t)$ constant for constant polarization. The LO is assumed to have constant power and a fixed frequency offset from the signal carrier frequency by $\delta \omega$. Thus,

$$
E_{1}=E_{1 x} \cos \left((\omega+\delta \omega) t+\varepsilon_{x}\right) \widehat{x}+E_{1 y} \cos \left((\omega+\delta \omega) t+\varepsilon_{y}\right) \widehat{y}
$$

In the heterodyne system of Figure 1, the total field incident on the detector is the vector sum of the signal and LO fields and is given by

$$
E_{t}=E_{s}+E_{1}
$$

Substituting (2-1) and (2-2) into (2-3) gives

$$
\begin{aligned}
\mathbf{E t}= & {\left[\mathrm{E}_{\mathrm{sx}}(\mathrm{t}) \cos \left(\omega \mathrm{t}+\theta(\mathrm{t})+\varepsilon_{s x}\right)+\mathrm{E}_{1 \mathrm{x}} \cos \left((\omega+\delta \omega) \mathrm{t}+\varepsilon_{1 \mathrm{x}}\right)\right] \widehat{\mathbf{x}} } \\
& +\left[\mathrm{E}_{\mathrm{sy}}(\mathrm{t}) \cos \left(\omega \mathrm{t}+\theta(\mathrm{t})+\varepsilon_{s x}\right)+\mathrm{E}_{\mathrm{ly}} \cos \left((\omega+\delta \omega) t+\varepsilon_{1 y}\right)\right] \widehat{\mathbf{y}}
\end{aligned}
$$

The detector current, $i_{d}$, is proportional to the time average of the field squared over an optical cycle. Thus,

$$
\begin{aligned}
i_{d} \sim<E t \cdot E t>= & \left(E_{s x}(t)^{2}+E_{l x}^{2}+E_{s y}(t)^{2}+E_{l y}^{2}\right) / 2 \\
& +E_{s x}(t) E_{l x} \cos \left(\delta \omega t-\theta(t)+\varepsilon_{l x}-\varepsilon_{s x}\right) \\
& +E_{s y}(t) E_{l y} \cos \left(\delta \omega t-\theta(t)+\varepsilon_{l y}-\varepsilon_{s y}\right) \\
= & i_{b b}+i_{s i g}
\end{aligned}
$$

where,

$$
i_{b b}=\left(E_{s x}(t)^{2}+E_{l x}^{2}+E_{s y}(t)^{2}+E_{l y}^{2}\right) / 2
$$




$$
\begin{aligned}
i_{\text {sig }}=E_{s x}(t) E_{1 x} \cos \left(\delta \omega t-\theta(t)+\varepsilon_{l x}-\varepsilon_{s x}\right) \\
+E_{s y}(t) E_{l y} \cos \left(\delta \omega t-\theta(t)+\varepsilon_{l y}-\varepsilon_{s y}\right)
\end{aligned}
$$

Equation (2-7) gives the baseband signal which is of no interest and will be be removed with a high pass filter. Equation (2-8) gives the signal of interest and has a carrier frequency of $\delta \omega$.

Thus, the total interference signal is a superposition of two IF signals with the magnitudes and relative phase determined by the polarizations of the beams. By inspection, it is clear that if the phase and magnitude for each term match, as is the case when the polarizations match, $i_{\text {sig }}$ will be maximum. On the other hand, if the beams are orthogonally polarized, the magnitudes will be identical, but the phase difference will be 180 degrees and $i_{\text {sig }}$ will be 0 . 


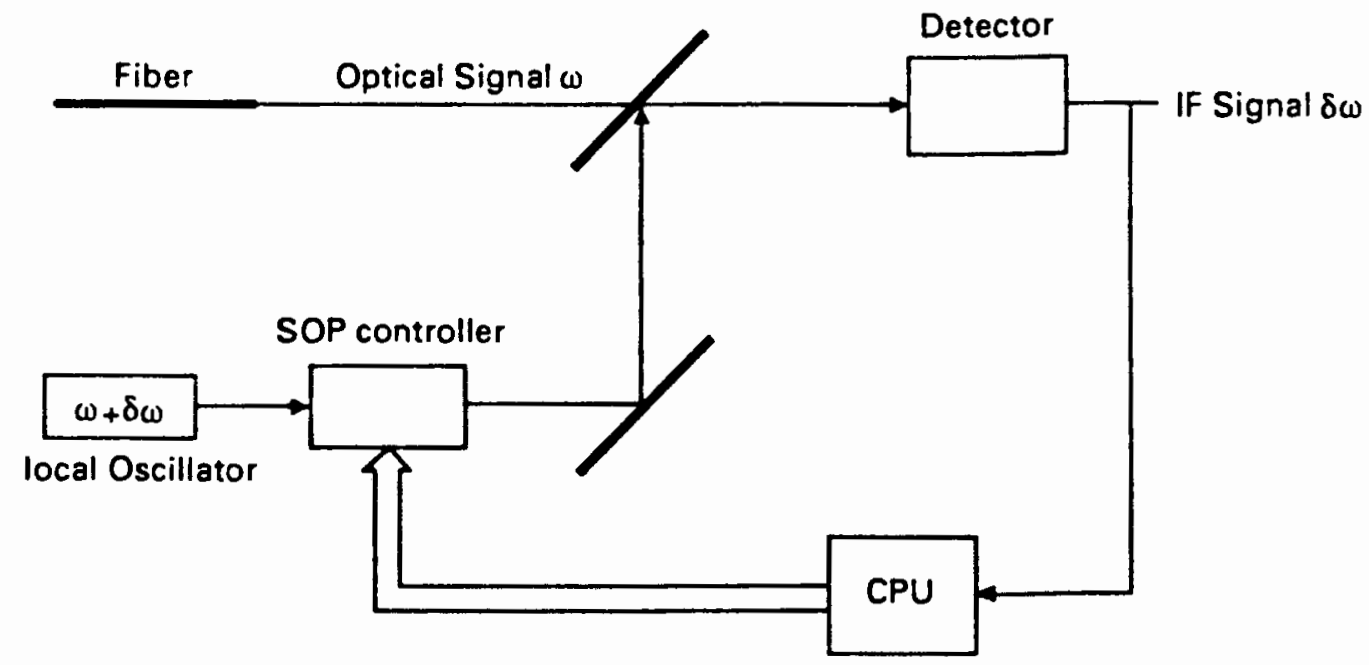

Figure 1. Implementation of an SOP matching system in fiber-optic heterodyne detection. 


\section{CHAPTER III}

\section{LIQUID CRYSTAL RETARDERS}

\section{INTRODUCTION}

Liquid crystals are long polymer molecules which under proper conditions, may collectively exist in mesophases. Mesophases are highly ordered fluid-like phases which cause the material to be highly anisotropic. Since this molecular ordering is determined by the physical environment, and may be distrorted by an electirc field, liquid crystals are useful for making field controlled variable birefringent devices [23].

The liquid crystals used in this work are aligned in the nematic mesophase in which the molecules align in the same direction. Each molecule experiences an elastic restoring force which tends to align it in the direction of its neighbor. Thus, the molecular ordering of the system may be chosen by placing the liquid crystals between two surfaces which are specially prepared to align the molecules at the boundary in the same direction. Two common methods for preparing the surfaces are rubbed polyimide and directionally sputtered $\mathrm{SiO}_{2}$. Either method will cause the molecules in contact with the surface to anchor in a uniform direction.

\section{LIQUID CRYSTAL RETARDERS}

A nematic liquid crystal retarder is made by introducing the liquid crystals between two plates of glass that have a transparent conductive 
coating of indium-tin-oxide (ITO) and a suitable surface treatment. The retardance, is a function of molecular alignment, which is controlled by an applied electric field and is given by

$$
\Gamma=\frac{2 \pi l}{\lambda}\left(n_{\theta}(V)-n_{d}\right)
$$

where $l$ is the cell thickness, $\lambda$ is the free-space wavelength, and $n_{e}(V)$ and $\mathrm{n}_{\mathrm{o}}$ are respectively the indices of refraction for the extraordinary and ordinary rays. The extraordinary index of refraction is a function of the applied voltage $\mathrm{V}$.

Figures 2 through 4 illustrate the operation of a variable liquid crystal retarder. In Figure 2, an electric field less than $V_{\text {th }}$ which is the threshold voltage required to overcome the molecular restoring forces. The molecular order is such that the retardance is maximum. Figure 3 shows the cell response when electric field is increased beyond $V_{t h}$. Distortion of the nematic order is greatest near the center since these molecules experience the weakest restoring forces. The rotation of these molecules reduces the retardance. In Figure 4, the applied electric field is equal to $\mathrm{V}_{\max }$, which is the breakdown voltage. The molecules on the surface are too tightly anchored to rotate completely in the direction of the field and the cell remains slightly birefringent.

Figure 5 is a graph of the retardance as a function of applied voltage measured at $633 \mathrm{~nm}$ for the liquid crystal retarders used in this work. The devices are made with ROTN 1132 liquid crystals and a $15.5 \mu \mathrm{m}$ spacing. Liquid crystal devices are addressed with a.c. voltages to prevent ion migration which degrades performance. By using different liquid crystals 
or a different cell spacing, the operating voltage range for the desired retardance range may be tailored to suit many applications. Generally, it is preferable to design the devices to operate in the region where $d \Gamma / \mathrm{dV}$ is small. Although this reduces the driving efficiency of the cells, here they are less sensitive to thermal fluctuations since the molecular restoring forces are dominant. 


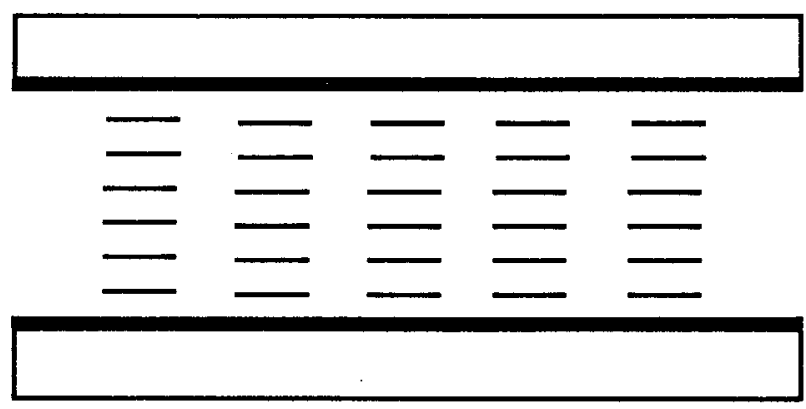

Figure 2. $\mathrm{V}<\mathrm{V}_{\text {th }}$

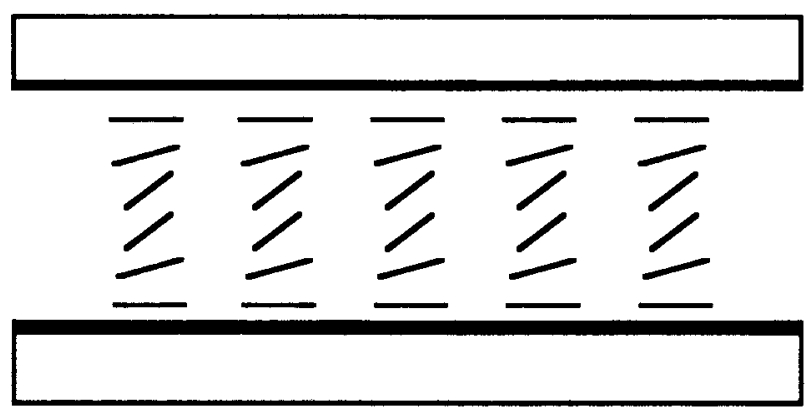

Figure 3. $\quad V_{\text {th }}<\mathrm{V}<\mathrm{V}_{\max }$

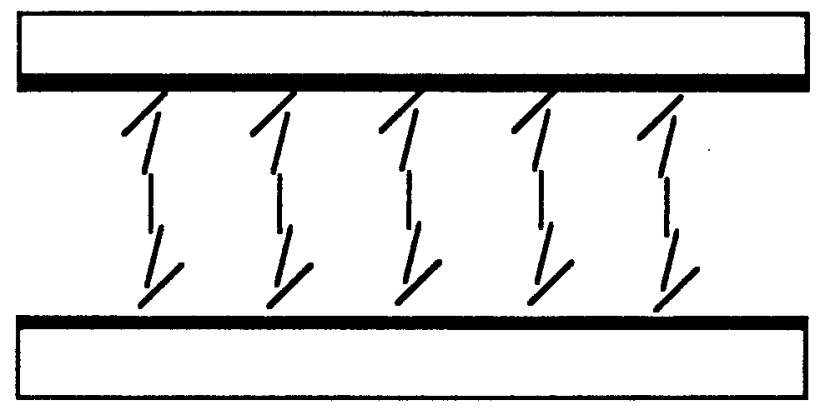

Figure 4. $\quad V=V \max$ 


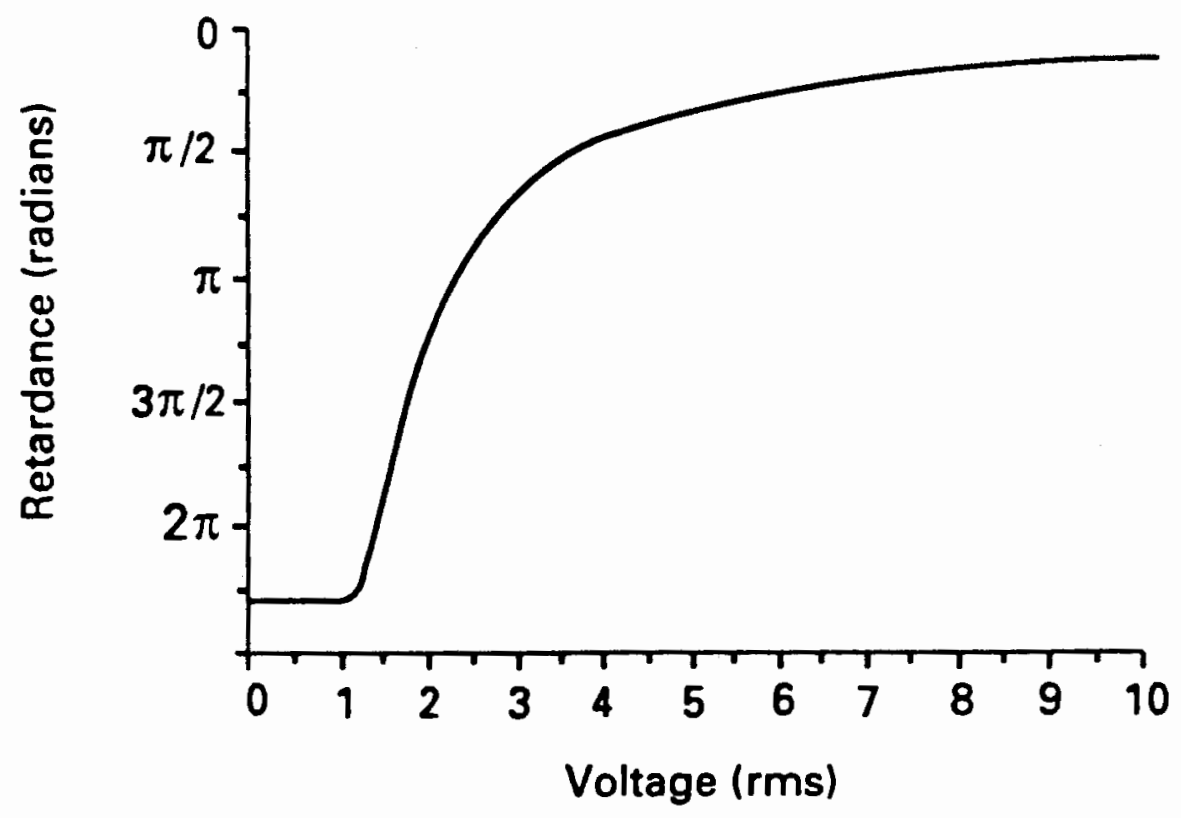

Figure 5. The retardance as a funciton of applied voltage for a typical liquid crystal retarder. 


\section{CHAPTER IV}

\section{SYSTEM OPERATION}

\section{INTRODUCTION}

The system we have developed is shown in figure 6. The LO is linearly polarized with the liquid crystal devices $d_{1}, d_{2}$ and $d_{3}$ tilted with their fast-axes at 45, 0 and 45 degrees respectively to the plane of LO polarization, which defines the $x$ axis of the coordinate system. $\Psi_{z}, \theta_{p}$ and $\theta_{y}$, which completely determine the output polarization, represent the retardances introduced by the three liquid crystal retarders and are functions of the applied voltages. The Jones vector describing the output polarization is obtained by multiplying the input polarization vector and the Jones matrices for each device in opposite order than they appear in the system, which gives

$$
\begin{aligned}
& \overrightarrow{\mathrm{E}}_{\text {out }}=\left[\begin{array}{cc}
\cos \left(\theta_{\mathrm{y}} / 2\right) & \mathrm{i} \sin \left(\theta_{\mathrm{y}} / 2\right) \\
\mathrm{i} \sin \left(\theta_{\mathrm{y}} / 2\right) & \cos \left(\theta_{\mathrm{y}} / 2\right)
\end{array}\right]\left[\begin{array}{cc}
\mathrm{e}^{\mathrm{i} \theta_{\mathrm{p}} / 2} & 0 \\
0 & \mathrm{e}^{-\mathrm{i} \theta_{\mathrm{p}} / 2}
\end{array}\right]\left[\begin{array}{cc}
\cos \left(\Psi_{\mathrm{z}} / 2\right) & \mathrm{i} \sin \left(\Psi_{\mathrm{z}} / 2\right) \\
\mathrm{i} \sin \left(\Psi_{\mathrm{z}} / 2\right) & \cos \left(\Psi_{\mathrm{z}} / 2\right)
\end{array}\right]\left[\begin{array}{l}
1 \\
0
\end{array}\right] \\
& =\left[\begin{array}{c}
\cos \frac{\theta_{\mathrm{p}}}{2} \cos \left(\frac{\Psi_{\mathrm{z}}+\theta_{\mathrm{y}}}{2}\right)+i \sin \frac{\theta_{\mathrm{p}}}{2} \cos \left(\frac{\Psi_{\mathrm{z}}-\theta_{\mathrm{y}}}{2}\right) \\
\sin \frac{\theta_{\mathrm{p}}}{2} \sin \left(\frac{\Psi_{\mathrm{z}}-\theta_{\mathrm{y}}}{2}\right)+i \cos \frac{\theta_{\mathrm{p}}}{2} \sin \left(\frac{\Psi_{\mathrm{z}}+\theta_{\mathrm{y}}}{2}\right)
\end{array}\right]
\end{aligned}
$$




\section{CONTROL ALGORITHM}

Two control algorithms are necessary to continuously and endlessly maintain the polarization match by appropriate adjustments to $\Psi_{\mathrm{z}}, \theta_{\mathrm{p}}$, and $\theta_{y}$. In the normal mode of operation, the controlling electronics systematically adjust the device voltages to maintain the maximum IF signal. These devices have a finite control range, so a reset mode is designed to bring the devices back into pre-defined operation limits while maintaining a constant output polarization.

\section{NORMAL MODE OF OPERATION}

The normal mode algorithm is designed to permit a system reset when necessary while maintaining the retardances within their operational ranges. If it is improperly designed, the system may enter a state from which no reset is possible. During the normal mode of operation, $d_{1}$ and $d_{2}$ operate as a single unit independent of $d_{3}$, and the output polarization is determined by two independently controlled units.

$\mathrm{d}_{1}$ and $\mathrm{d}_{2}$ operate as a linear state rotator which rotates the polarization at point $\mathbf{B}$ (see Figure 6) endlessly in either direction and which will be referred to as the rotator analog hereafter. The polarization at point $\mathbf{B}$ is given by

$$
\overrightarrow{\mathrm{E}_{\mathrm{B}}}=\left[\begin{array}{c}
\cos \frac{\Psi_{\mathrm{z}}}{2} \\
\mathrm{e}^{\mathrm{i}\left(\pi / 2-\theta_{\mathrm{p}}\right) \sin \frac{\Psi_{\mathrm{z}}}{2}}
\end{array}\right]
$$


where the absolute phase term has been ignored since it has no effect on the polarization. Note that if $\Psi_{z}=2 n \pi(n=0, \pm 1, \pm 2, \ldots)$, the polarization is

$$
\overrightarrow{\mathrm{E}_{\mathrm{B}}}=\left[\begin{array}{l}
1 \\
0
\end{array}\right]
$$

and when $\Psi_{Z}=(2 n+1) \pi$, the polarization is

$$
\overrightarrow{\mathrm{E}_{\mathrm{B}}}=\left[\begin{array}{l}
0 \\
1
\end{array}\right]
$$

Since the eigenstates of $d_{2}$ are aligned to the coordinate axes, the polarization at point $\mathbf{A}$ matches one of the two eigenstates of $\mathrm{d}_{2}$ when $\Psi_{\mathrm{Z}}=$ $\mathrm{n} \pi$, and changes in $\theta_{\mathrm{p}}$ have no effect on the polarization at point $\mathbf{B}$. It will be shown that the polarization at point $\mathbf{B}$ may be rotated endlessly by choosing the operating range limits of $\Psi_{Z}$ to be any integral multiples of $\pi$, and by switching between appropriate values of $\theta_{p}$ at these range limits.

Now, if $\theta_{p}=(4 n+1) \pi / 2$ the polarization at $\mathbf{B}$ is

$$
\overrightarrow{\mathrm{E}_{\mathrm{B}}}=\left[\begin{array}{c}
\cos \left(\Psi_{\mathrm{z}} / 2\right) \\
\sin \left(\Psi_{\mathrm{z}} / 2\right)
\end{array}\right]
$$

which is linear with the plane of polarization tilted $\Psi_{Z} / 2$. Similarly, for $\theta_{\mathrm{p}}=(4 \mathrm{n}-1) \pi / 2$

$$
\overrightarrow{\mathrm{E}}_{\mathrm{B}}=\left[\begin{array}{c}
\cos \left(\Psi_{\mathrm{z}} / 2\right) \\
-\sin \left(\Psi_{\mathrm{z}} / 2\right)
\end{array}\right]
$$


which has a tilt of $-\Psi_{\mathrm{Z}} / 2$. In the normal mode of operation, $\theta_{\mathrm{p}}$ is set to either $(4 \mathrm{n} \pm 1) \pi / 2$ and the polarization at point $\mathbf{B}$ is linear. Thus, the tilt angle at point $\mathbf{B}$ may be rotated endlessly in either direction by reversing the direction of control of $\Psi_{Z}$ at the boundary of its range limit and switching $\theta_{\mathrm{p}}$ to its complimentary value. At these transition points, the polarization will be given by (4-3) or (4-4) depending on which range limit has been reached.

For complete polarization control $\mathrm{d}_{3}$ is required. The polarization control of the entire system can be represented as two rotations about orthogonal axes on the Poincare sphere as illustrated in Figure 7. Since $\theta_{p}$ $=(4 \mathrm{n} \pm 1) \pi / 2$, the rotator analog causes a rotation about the $\mathrm{Z}-\mathrm{Z}^{*}$ axis along the equator by an angle $\pm \Psi_{Z}$, while $\mathrm{d}_{3}$ causes a rotation about the $\theta_{y}-\theta_{y} *$ axis by an angle $\theta_{y}$. Clearly, all possible polarization states may be matched with these independent rotations.

\section{RESET MODE OF OPERATION}

Since the rotator analog operates endlessly, no reset of $d_{1}$ and $d_{2}$ is necessary. However, $d_{3}$ will periodically reach a range limit and a reset of the entire system will be required. No polarization tracking is possible during the reset mode so it is important that the reset is completed in a time short compared to the rate of change of SOP in the signal fiber. A system built with standard liquid crystal devices is capable of a reset in less than a second. This is far faster than the required reset interval since the SOP change in installed fiberoptic networks is on the order of hours or days [6]. 
During the reset cycle, $d_{1}$ and $d_{2}$ no longer operate as a rotator and the polarization at point $\mathbf{B}$ will not be linear. As $\mathrm{d}_{3}$ is brought back into its operational range, $\mathrm{d}_{1}$ and $\mathrm{d}_{2}$ are controlled in a manner that exactly compensates for the changes in $d_{3}$ so that the output SOP remains constant.

The reset cycle is initiated when $\theta_{\mathrm{y}}$ reaches its upper or lower range limits. In general, these limits may be set to any integral multiple of $\pi$, however, for clarity, only the limits of 0 and $\pi$ are discussed here. Similarly, the operational limits of $\Psi_{Z}$ and $\theta_{p}$ are chosen to be 0 to $\pi$ and 0 to $2 \pi$ respectively. Thus, when the upper bound reset cycle is initiated, $\theta_{y}$ $=\pi, \theta_{\mathrm{p}}=\pi / 2$ or $3 \pi / 2$, and $\Psi_{\mathrm{Z}}=\Psi_{\mathrm{zo}}$, where $0 \leq \Psi_{\mathrm{zo}} \leq \pi$, and according to (4-1), the polarization will be

$$
\overrightarrow{\mathrm{E}}_{\text {reset }}=\left[\begin{array}{c}
\cos \left(\Psi_{\mathrm{zo}} / 2\right) \\
\pm \sin \left(\Psi_{\mathrm{zo}} / 2\right)
\end{array}\right]
$$

Similarly, when the lower range reset is initiated $\theta_{y}=0, \theta_{p}=\pi / 2$ or $3 \pi / 2$, and $\Psi_{\mathrm{Z}}=\Psi_{\mathrm{zo}}$, where $0 \leq \Psi_{\mathrm{zo}} \leq \pi$, and the polarization will be

$$
\overrightarrow{\mathrm{E}}_{\text {reset }}=\left[\begin{array}{c}
\sin \left(\Psi_{\mathrm{zo}} / 2\right) \\
\pm \cos \left(\Psi_{\mathrm{zo}} / 2\right)
\end{array}\right]
$$

The reset conditions on $\theta_{\mathrm{y}}, \theta_{\mathrm{p}}$ and $\Psi_{\mathrm{z}}$ are derived below using (4-1) and assuming a constant polarization given by (4-7). For the upper range reset,

$$
\begin{aligned}
& \theta_{\mathrm{y}}=\pi-\operatorname{Cos}^{-1}\left(\cos \Psi_{\mathrm{z}} / \cos \Psi_{\mathrm{zo}}\right) \\
& \theta_{\mathrm{p}}=\theta_{\mathrm{po}}-\mathrm{MCos}^{-1}\left(\sin \Psi_{\mathrm{zo}} / \sin \Psi_{\mathrm{z}}\right)
\end{aligned}
$$


where

$$
\begin{array}{clc}
0 \leq \operatorname{Cos}^{-1}(\mathrm{x}) \leq \pi & \\
\mathrm{M}=1 \quad \text { if } & 0 \leq \Psi_{\mathrm{zo}}<\pi / 2 \text { and } \theta_{\mathrm{po}}=\pi / 2 \\
& \text { or } \\
& \pi / 2 \leq \Psi_{\mathrm{zo}}<\pi \text { and } \theta_{\mathrm{po}}=3 \pi / 2
\end{array}
$$

and

$M=-1 \quad$ if

$0 \leq \Psi_{\mathrm{zo}}<\pi / 2$ and $\theta_{\mathrm{po}}=3 \pi / 2$

or

$\pi / 2 \leq \Psi_{\mathrm{zo}}<\pi$ and $\theta_{\mathrm{po}}=\pi / 2$

where $\theta_{\mathrm{po}}$ and $\Psi_{\mathrm{zo}}$ are the initial values of $\theta_{\mathrm{p}}$ and $\Psi_{\mathrm{z}}$. When $\theta_{\mathrm{y}}$ reaches its lower range limit,

$$
\begin{aligned}
& \theta_{\mathrm{y}}=\operatorname{Cos}^{-1}\left(\cos \Psi_{\mathrm{z}} / \cos \Psi_{\mathrm{zo}}\right) \\
& \theta_{\mathrm{p}}=\theta_{\mathrm{po}}+\mathrm{MCos}^{-1}\left(\sin \Psi_{\mathrm{zo}} / \sin \Psi_{\mathrm{z}}\right)
\end{aligned}
$$

where $\mathrm{M}$ is determined by the same relationships above.

During the reset, the values of $\theta_{y}$ and $\theta_{p}$ are calculated according to (4-8) and (4-9) or (4-10) and (4-11) as $\Psi_{Z}$ is incrementally changed from $\Psi_{\mathrm{ZO}}$ to $\pi-\Psi_{\mathrm{ZO}}$. When the cycle is completed, $\operatorname{Cos}^{-1}\left(\cos \Psi_{\mathrm{Z} /} \cos \Psi_{\mathrm{ZO}}\right)=\pi$ and $\operatorname{Cos}^{-1}\left(\sin \Psi_{z o} / \sin \Psi_{z}\right)=0$, so that $\theta_{\mathrm{p}}=\theta_{\mathrm{po}}$, but $\theta_{\mathrm{y}}=0$ and $\pi$ for the upper and lower limit resets respectively. 


\section{Derivation of Upper Range Reset}

The upper range reset algorithm is derived by solving (4-1) for the magnitude and phase of the $\mathrm{x}$ and $\mathrm{y}$ components and setting them equal to the terms of $(4-7 a)$. Thus,

$$
\begin{aligned}
& A_{x}{ }^{2}=\cos ^{2} P \cos ^{2}(Z+Y)+\sin ^{2} P \cos ^{2}(Z-Y)=\sin ^{2} Z_{o} \\
& A_{y}{ }^{2}=\sin ^{2} P \sin ^{2}(Z-Y)+\cos ^{2} P \sin ^{2}(Z+Y)=\cos ^{2} Z_{o} \\
& \varepsilon_{x}-\varepsilon_{y}=\tan ^{-1}\left(\tan P \frac{\cos (Z-Y)}{\cos (Z+Y)}\right)-\tan ^{-1}\left(\cot P \frac{\sin (Z+Y)}{\sin (Z-Y)}\right)=n \pi
\end{aligned}
$$

where

$$
\begin{aligned}
& \mathrm{Z}=\Psi_{\mathrm{Z}} / 2 \\
& \mathrm{Z}_{\mathrm{o}}=\Psi_{\mathrm{Zo}} / 2 \\
& \mathrm{P}=\theta_{\mathrm{p}} / 2 \\
& \mathrm{Y}=\theta_{\mathrm{y}} / 2 \\
& \mathrm{n}=0, \pm 1, \pm 2, \ldots
\end{aligned}
$$

and $A_{x}$ and $A_{y}$ are the magnitudes of the $x$ and $y$ components and $\varepsilon_{x}$ and $\varepsilon_{y}$ are the phase terms. Conditions (4-12) and (4-13) ensure that the magnitude of the field components remain constant, and (4-14) requires the polarization to remain linear.

By (4-14),

$$
\tan ^{-1}\left(\tan P \frac{\cos (Z-Y)}{\cos (Z+Y)}\right)=\tan ^{-1}\left(\cot P \frac{\sin (Z+Y)}{\sin (Z-Y)}\right)+n \pi
$$




$$
\begin{aligned}
\tan P \frac{\cos (Z-Y)}{\cos (Z+Y)} & =\tan \left\{\tan ^{-1}\left(\cot P \frac{\sin (Z+Y)}{\sin (Z-Y)}\right)+n \pi\right\} \\
& =\tan \left\{\tan ^{-1}\left(\cot P \frac{\sin (Z+Y)}{\sin (Z-Y)}\right)\right\} \\
& =\cot P \frac{\sin (Z+Y)}{\sin (Z-Y)}
\end{aligned}
$$

The previous expression is valid even if the $\tan ^{-1}$ function is restricted to principal values since any discrepancy is absorbed in the $n \pi$ term. Dividing both sides of the last expression by cotP gives

$$
\begin{aligned}
\tan ^{2} \mathrm{P} & =\frac{\cos (\mathrm{Z}+\mathrm{Y}) \sin (\mathrm{Z}+\mathrm{Y})}{\cos (\mathrm{Z}-\mathrm{Y}) \sin (\mathrm{Z}-\mathrm{Y})} \\
& =\frac{\sin (2 \mathrm{Z}+2 \mathrm{Y})}{\sin (2 \mathrm{Z}-2 \mathrm{Y})}
\end{aligned}
$$

Using the identity,

$$
\tan ^{2} \alpha=\frac{1-\cos 2 \alpha}{1+\cos 2 \alpha}
$$

and solving for $\cos 2 \mathrm{P}$ gives

$$
\cos 2 \mathrm{P}=\frac{1-\frac{\sin (2 Z+2 Y)}{\sin (2 Z-2 Y)}}{1+\frac{\sin (2 Z+2 Y)}{\sin (2 Z-2 Y)}}
$$




$$
\begin{aligned}
& =\frac{\sin (2 Z-2 Y)-\sin (2 Z+2 Y)}{\sin (2 Z-2 Y)+\sin (2 Z+2 Y)} \\
& =\frac{-2 \cos 2 Z \sin 2 Y}{2 \sin 2 Z \cos 2 Y}
\end{aligned}
$$

Thus,

$$
\cos 2 \mathrm{P}=-\cot 2 \mathrm{Ztan} 2 \mathrm{Y}
$$

Taking (4-13) minus (4-12) and using the identity,

$$
\cos 2 \alpha=\cos ^{2} \alpha-\sin ^{2} \alpha
$$

gives

$$
\begin{aligned}
\cos 2 Z_{0}= & \sin ^{2} P \sin ^{2}(Z-Y)+\cos ^{2} P \sin ^{2}(Z+Y) \\
& \quad-\cos ^{2} P \cos ^{2}(Z+Y)-\sin ^{2} P \cos ^{2}(Z-Y) \\
= & \sin ^{2} P\left[\sin ^{2}(Z-Y)-\cos ^{2}(Z-Y)\right] \\
& \quad+\cos ^{2} P\left[\sin ^{2}(Z+Y)-\cos ^{2}(Z+Y)\right] \\
= & \sin ^{2} P[-\cos (2 Z-2 Y)]+\cos ^{2} P[-\cos (2 Z+2 Y] \\
= & -\sin ^{2} P[\cos 2 Z \cos 2 Y+\sin 2 Z \sin 2 Y] \\
& \quad+\cos ^{2} P[\sin 2 Z \sin 2 Y-\cos 2 Z \cos 2 Y] \\
= & -\cos 2 Z \cos 2 Y\left[\sin ^{2} P+\cos ^{2} P\right]+\sin 2 Z \sin 2 Y\left[\cos ^{2} P-\sin ^{2} P\right]
\end{aligned}
$$

Thus, 


$$
\cos 2 Z_{\mathrm{o}}=-\cos 2 \mathrm{Z} \cos 2 \mathrm{Y}+\cos 2 \mathrm{P} \sin 2 \mathrm{Z} \sin 2 \mathrm{Y}
$$

Substituting (4-16) into (4-17),

$$
\begin{aligned}
\cos 2 Z_{0} & =-\cos 2 Z \cos 2 Y-\cot 2 Z \tan 2 Y \sin 2 z \sin 2 Y \\
& =\frac{-\cos 2 Z \cos ^{2} 2 Y-\cos 2 Z \sin ^{2} 2 Y}{\cos 2 Y} \\
& =\frac{-\cos 2 Z\left[\cos ^{2} 2 Y+\sin ^{2} 2 Y\right]}{\cos 2 Y} \\
& =\frac{-\cos 2 Z}{\cos 2 Y}
\end{aligned}
$$

Thus,

$$
\cos 2 Y=\frac{-\cos 2 Z}{\cos 2 Z_{o}}
$$

Since $\theta_{y}=2 Y$ is defined to be in the range 0 to $\pi$, the principal value identity,

$$
\operatorname{Cos}^{-1}(-\mathrm{x})=\pi-\operatorname{Cos}^{-1} \mathrm{x} \quad 0 \leq \operatorname{Cos}^{-1} \mathrm{x} \leq \pi
$$

gives,

$$
2 \mathrm{Y}=\pi-\operatorname{Cos}^{-1}\left(\frac{\cos 2 \mathrm{Z}}{\cos 2 \mathrm{Z}_{0}}\right)
$$


Substituting (4-15a), (4-15b), and (4-15d) gives,

$$
\theta_{y}=\pi-\operatorname{Cos}^{-1}\left(\frac{\cos \Psi_{z}}{\cos \Psi_{z 0}}\right)
$$

which confirms (4-8) in the text.

The conditions on $\theta_{p}$ are derived below. Substituting (4-17) into (415) yields,

$$
\begin{aligned}
\cos 2 \mathrm{P} & =\frac{\cos 2 Z_{\cos 2 Z_{\alpha} \sin 2 Y}}{\sin 2 Z_{\cos } 2 Z} \\
& =\frac{\cos 2 Z_{\alpha} \sin 2 Y}{\sin 2 Z}
\end{aligned}
$$

Thus,

$$
\sin 2 Y=\frac{\sin 2 Z \cos 2 P}{\cos 2 Z_{o}}
$$

Substituting (4-18) and (4-21) into (4-17),

$$
\cos 2 Z_{0}=\frac{\cos ^{2} 2 Z+\cos ^{2} 2 P \sin ^{2} 2 Z}{\cos 2 Z_{o}}
$$

Thus,

$$
\cos ^{2} 2 Z_{o}=\cos ^{2} 2 Z+\cos ^{2} 2 P \sin 22 Z
$$




$$
\begin{aligned}
\cos ^{2} 2 \mathrm{P} & =\frac{\cos ^{2} 2 Z_{0}-\cos ^{2} 2 Z}{\sin ^{2} 2 Z} \\
& =\frac{\cos ^{2} 2 Z_{0}-\sin ^{2} 2 Z-1}{\sin ^{2} 2 Z} \\
& =\frac{-\sin ^{2} 2 Z_{0}-\sin ^{2} 2 Z}{\sin ^{2} 2 Z} \\
& =\frac{-\sin ^{2} 2 Z_{0}}{\sin ^{2} 2 Z}+1
\end{aligned}
$$

$$
\cos ^{2} 2 P-1=\frac{-\sin ^{2} 2 Z_{0}}{\sin ^{2} 2 Z}
$$$$
\sin ^{2} 2 P=\frac{\sin ^{2} 2 Z_{0}}{\sin ^{2} 2 Z}
$$$$
\pm \sin 2 \mathrm{P}=\frac{\sin 2 \mathrm{Z}_{\mathrm{o}}}{\sin 2 \mathrm{Z}}
$$$$
2 P=\sin ^{-1}\left(\frac{ \pm \sin 2 Z_{0}}{\sin 2 Z}\right)
$$

Principal values of $\sin ^{-1} x$ are in the range $\pm \pi / 2$. Since $\theta_{p}=2 P$ might not be in this range during the reset cycle, two cases must beconsidered. For principal values of $2 \mathrm{P}$ we can use the identity,

$$
\operatorname{Sin}^{-1} \mathrm{x}=\pi / 2-\operatorname{Cos}^{-1} \mathrm{x}
$$

$$
0 \leq \operatorname{Cos}^{-1} \mathrm{x} \leq \pi
$$




$$
-\pi / 2 \leq \operatorname{Sin}^{-1} \mathrm{x} \leq \pi / 2
$$

and substitute (4-15a), (4-15b), (4-15c) which yields,

$$
\theta_{\mathrm{p}}=\frac{\pi}{2}-\operatorname{Cos}^{-1}\left(\frac{\sin \Psi_{\mathrm{zo}}}{\sin \Psi_{\mathrm{z}}}\right)
$$

This is (4-9) for $0 \leq \Psi_{\mathrm{zo}} \leq \pi / 2$ and $\theta_{\mathrm{po}}=\pi / 2$. Also by (4-22),

$$
\theta_{\mathrm{p}}=\frac{\pi}{2}-\operatorname{Cos}^{-1}\left(\frac{-\sin \Psi_{\mathrm{zo}}}{\sin \Psi_{\mathrm{z}}}\right)
$$

Using (4-19), (4-25) becomes,

$$
\theta_{p}=-\frac{\pi}{2}+\operatorname{Cos}^{-1}\left(\frac{\sin \Psi_{z o}}{\sin \Psi_{z}}\right)
$$

Since a retardance of $-\pi / 2$ radians has the identical effect on polarization as $3 \pi / 2$ radians, (4-26) confirms (4-9) for $0 \leq \Psi_{\mathrm{zo}} \leq \pi / 2$ and $\theta_{\mathrm{po}}=3 \pi / 2$. When $\pi / 2>\Psi_{\mathrm{zo}} \leq \pi$, the non-principal values of (4-22) are used which gives,

$$
2 \mathrm{P}=\pi-\operatorname{Sin}^{-1}\left(\frac{ \pm \sin 2 \mathrm{Z}_{\mathrm{o}}}{\sin 2 \mathrm{Z}}\right)
$$

where the principal valued function, $\mathrm{Sin}^{-1}$, function will return values in the range $-\pi / 2$ to $\pi / 2$. Again, using (4-23) and substituting (4-15a), (4-15b), and (4-15c), (4-27) becomes, 


$$
\theta_{p}=\frac{\pi}{2}+\operatorname{Cos}^{-1}\left(\frac{\sin \Psi_{z o}}{\sin \Psi_{z}}\right)
$$

which is (4-9) for $\pi / 2 \leq \Psi_{z o} \leq \pi$, and $\theta_{\text {po }}=\pi / 2$. Using (4-19) for the negative argument in (4-27) gives,

$$
\theta_{\mathrm{p}}=-\frac{\pi}{2} \cdot \cos ^{-1}\left(\frac{\sin \Psi_{\mathrm{zo}}}{\sin \Psi_{\mathrm{z}}}\right)
$$

which confirms (4-9) for $\pi / 2 \leq \Psi_{\mathrm{zo}} \leq \pi$, and $\theta_{\mathrm{po}}=3 \pi / 2$.

\section{Derivation of Lower Range reset}

The lower range reset can be derived by reversing the process of the upper range reset. The initial and final values of $\theta_{y}$ for the upper range reset are exactly the final and inital values respectively for the lower range reset. Thus, subracting (4-8) from $\pi$ gives (4-10). Similarly, (4-11) follows by switching the inital conditions on (4-9). In order to prevent continual swithching between the upper and lower reset modes, a slight hysteresis must be programmed into the controlling system. 


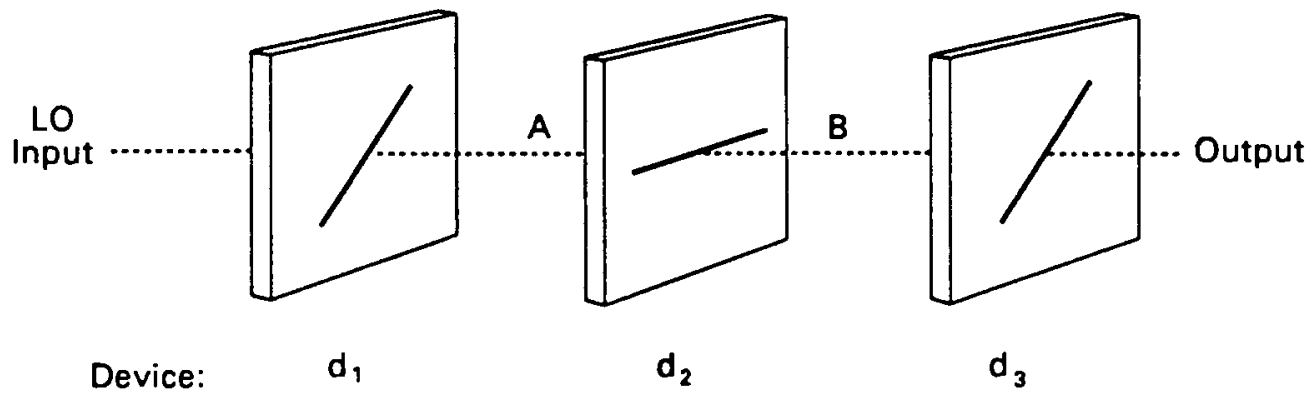

Tilt (degrees):

45

0

45

Retardance:

$\psi_{z}$

$\theta_{\dot{p}}$

$\theta_{Y}$

Figure 6. The SOP matching system showing the orientation and retardance of the liquid crystal devices.

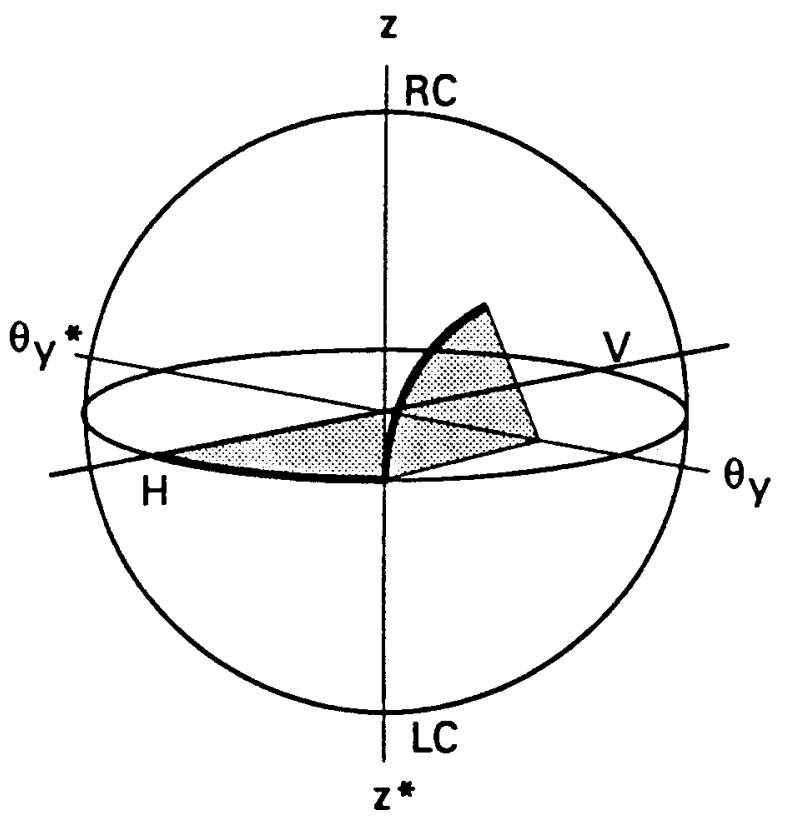

Figure 7. A Poincare sphere representation of SOP matching during the normal mode of operation. 


\section{CHAPTER V}

\section{EXPERIMENTAL VERIFICATION}

\section{INTRODUCTION}

The operation of the system is verified with two experiments. One illustrates the endless control of the rotator analog while the other demonstrates the normal and reset modes of the entire system. The experiments are designed to demonstrate the operation of the polarization controller, and are not intended to mimic an actual heterodyning system.

\section{EXPERIMENTAL APPARATUS}

The experimental apparatus is shown in Figure 8. A polarized $\mathrm{HeNe}$ laser is used as a source with the plane of polarization defined to be 0 degrees. Devices $d_{1}, d_{2}$, and $d_{3}$ are liquid crystal retarders and are mounted on rotational mounts which rotate in the plane normal to the laser beam. The a.c voltages $\mathrm{V}_{\mathrm{d} 1}, \mathrm{~V}_{\mathrm{d} 2}$, and $\mathrm{V}_{\mathrm{d} 3}$ are controlled independently. A polarizer which is also mounted on a rotational mount, and a power meter are used to determine the output polarization by rotating the polarizer and measuring the transmitted power. The angle of maximum transmission gives the tilt, and the ellipticity is defined as the minimum transmission over the maximum transmission. 


\section{CHARACTERIZATION OF RETARDERS}

Before beginning the experiments, the rotational mounts must be calibrated in reference to the fast axes of the retarders so that the rotation angle may be known accurately. Then the retardance characteristics for each cell must be measured.

Initially, the retarders are removed and the polarizer is rotated to 90 degrees for maximun extinction so that no transmitted power is measured. Any device placed in front of the polarizer which changes the polarization will cause light to transmit through the polarizer which will be measured with the power meter. Since a linearly polarized beam remains linear when it propagates in one of the two eigen-axes of a retarder, the fast and slow-axes of each retarder can be located by inserting it in the beam and rotating it until no transmitted light is detected. The fast-axis is the one measured to be orthogonal to the liquid crystal alignment direction.

The retardance of the cell is measured by rotating the fast axis of the retarder to 45 degrees with the polarizer still at 90 degrees. This is a standard configuration for an electro-optic modulator and the transmitted power can be shown to be [24]

$$
\mathrm{P}_{\text {out }}=\mathrm{P}_{\mathrm{in}} \sin ^{2} \Gamma(\mathrm{V})
$$

where $P_{\text {in }}$ is the input optical power, $\Gamma(V)$ is the retardance which is a function of applied voltage, and $\mathrm{P}_{\text {out }}$ is the transmitted power. Hence, 
$\Gamma(v)=\sin ^{-1} \sqrt{\frac{P_{\text {out }}}{P_{\text {in }}}}$

By slowly increasing the volage across the cell, the transmitted power will go through several minima and maxima every $\pi / 2$ of retardance until there is little or no change near a minimum. Here the cell has a retardance close to zero and increasing the voltage much beyond this point will cause the cell to break down. These measurements were made for each cell and were found to be nearly identical. The retardance curve for a typical cell is plotted in Figure 5.

\section{ROTATOR ANALOG}

The first experiment demonstrates the endless control of the rotator analog. Devices $\mathrm{d}_{1}$ and $\mathrm{d}_{2}$ are placed in the experimental apparatus with a 45 and 0 degree tilt respectively. Initially, the drive voltages to $d_{1}$ and $d_{2}$ $\left(\mathrm{V}_{\mathrm{d} 1}\right.$, and $\left.\mathrm{V}_{\mathrm{d} 2}\right)$ are set equal to $1.31 \mathrm{~V}$, and $1.72 \mathrm{~V}$ to provide $2 \pi$ and $3 \pi / 2$ radians of phase retardance respectively, and the polarization at point $\mathbf{B}$ is measured to be linear and to have a 0 degree tilt. $V_{d 1}$ is then increased which reduces $\Psi_{\mathrm{z}}$, and a positive rotation of the polarization results. When $\mathrm{V}_{\mathrm{d} 1}$ is $2.32 \mathrm{~V}, \Psi_{\mathrm{Z}}=\pi$, and $\mathrm{V}_{\mathrm{d} 2}$ is increased to $3.60 \mathrm{~V}$ which gives $\theta_{\mathrm{p}}=$ $\pi / 2$. During this transition, the polarization is measured to remain linear and to have a constant tilt of 90 degrees. Now, as $\mathrm{V}_{\mathrm{d} 1}$ is decreased, polarization rotation continues in the positive direction until a rotation of 180 degrees has been reached. Once again $V_{d 1}$ is $1.31 \mathrm{~V}$ and $\Psi_{z}=2 \pi$ so that the polarization at the input to $\mathrm{d}_{2}$ matches one of the eigen states of that device and $V_{d}$ may be switched back to $1.72 \mathrm{~V}$ so that $\theta_{\mathrm{p}}=3 \pi / 2$. 
Thus, the rotation will continue in the same direction by increasing $V_{d 1}$. Experimental plots of $\mathrm{V}_{\mathrm{d} 1}$ and $\mathrm{V}_{\mathrm{d} 2}$ for a 180 degree rotation are given in Figure 9 for every 10 degree increment.

\section{SYSTEM DEMONSTRATION}

The second experiment demonstrates the operation of the entire system for both the normal and reset modes. Now all three liquid crystral retarders are in place with the orientation described in Chapter IV. Initially, $\mathrm{V}_{\mathrm{d} 1}, \mathrm{~V}_{\mathrm{d} 2}$ and $\mathrm{V}_{\mathrm{d} 3}$ are set to $1.31 \mathrm{~V}, 1.72 \mathrm{~V}, 10 \mathrm{~V}$, so that $\Psi_{\mathrm{z}}=$ $2 \pi, \theta_{p}=3 \pi / 2$, and $\theta_{y}=0$, and the polarization is measured to be linear with a 0 degree tilt. The drive voltages are now systematically adjusted to produce polarizations which map onto the Poincare sphere as a locus of points indicated in Figure 10. A reset cycle is required when $\theta_{y}=\pi$.

The contolling voltages for the 12 polarization states and for 6 intermediate states of the reset cycle are plotted in Figure 11. The voltages of points 1 through 6 correspond to the first six polarization states starting with the $\mathbf{H}$ polarization which is horizontal and linear. Voltages 7 through 11 demonstrate the reset cycle and are indicated in the shaded region. Here the polarization is maintained at $\mathbf{V}$ which is vertical and linear. The normal mode of operation is continued with voltages 12 through 18 , which brings the polarization back to $\mathbf{H}$. This demonstrates the endless control possible by the system when a reset is done at the appropriate time. 


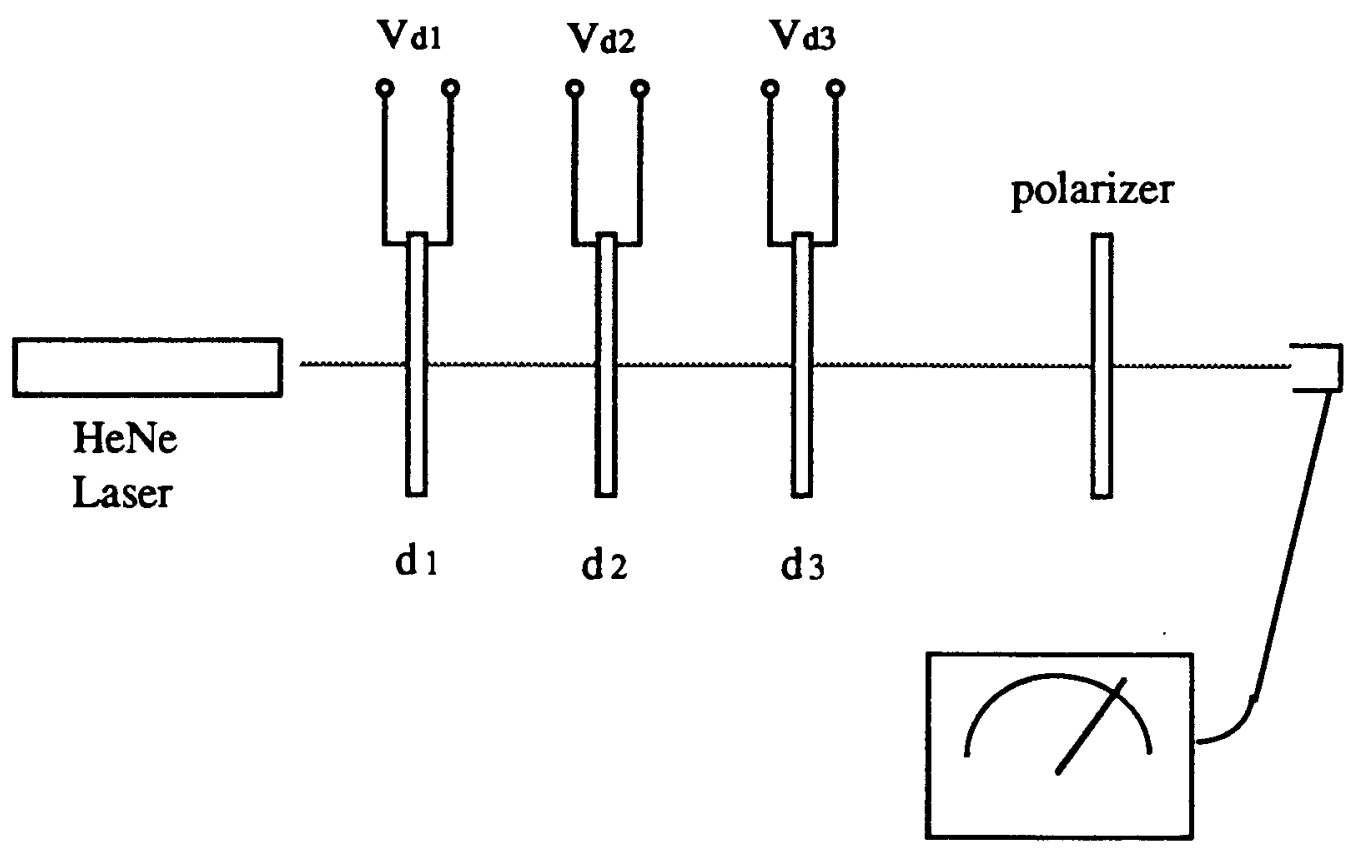

Power meter

Figure 8. Experimental apparatus.

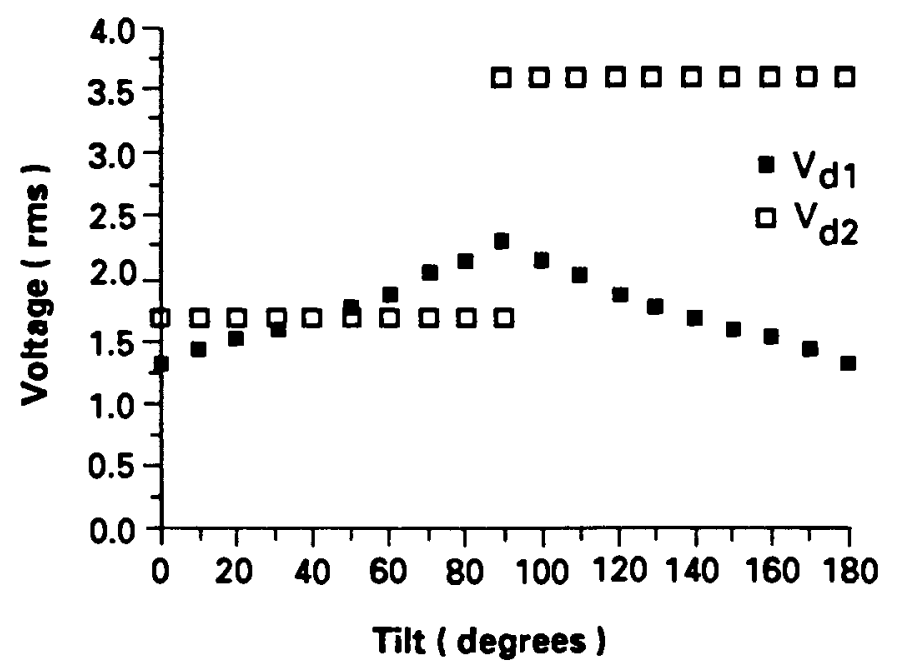

Figure 9. A plot of Vd1 and Vd2 for several tilt angles of the polarizations. 


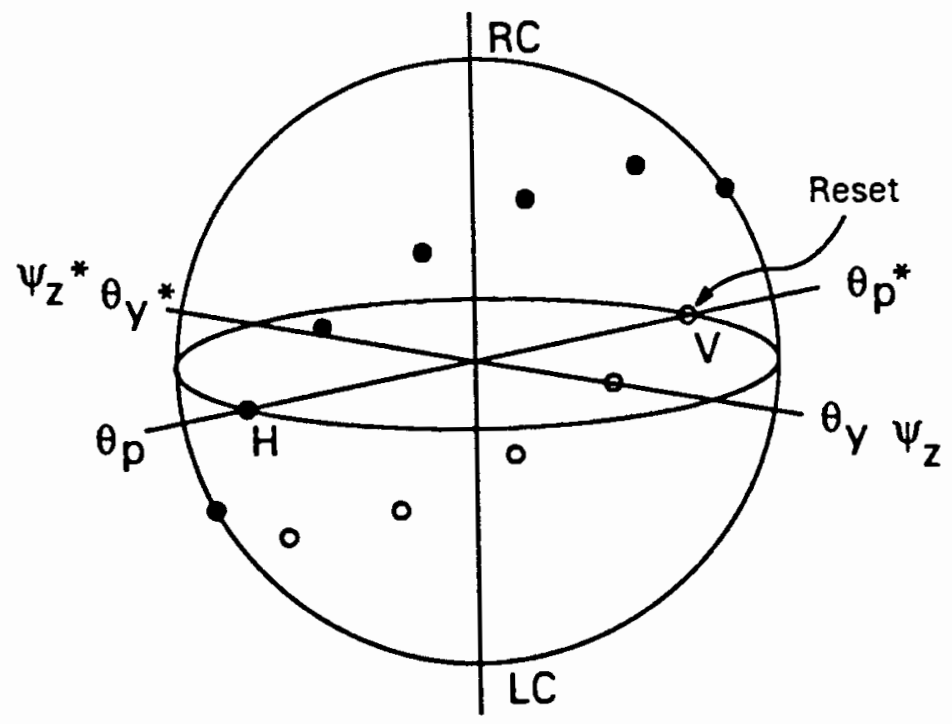

Figure 10. A plot of the polarization states used in demonstrating endless and complete polarization control. 

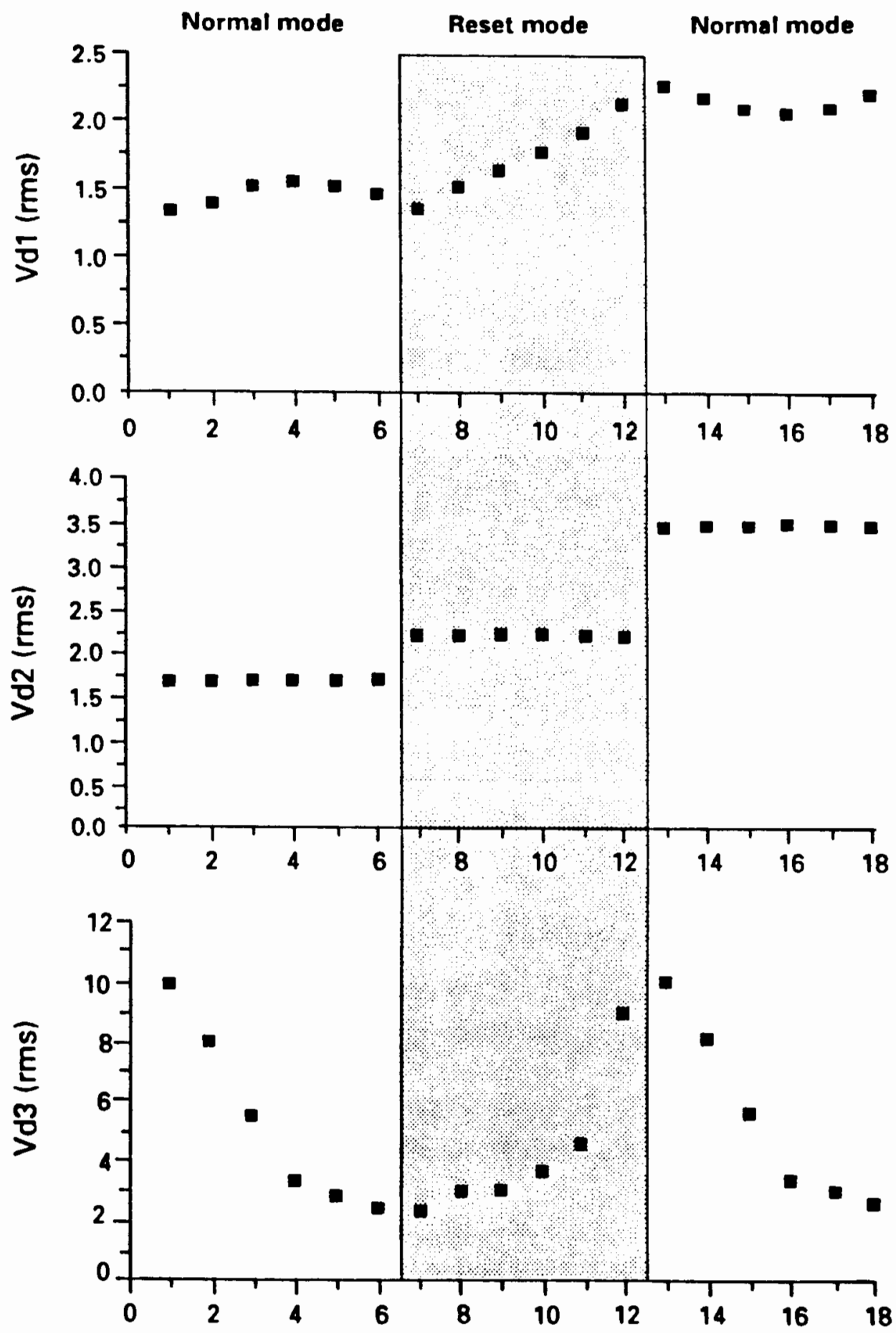

Figure 11. A plot of the control voltages demonstrating normal and reset modes. 


\section{CHAPTER VI}

\section{CONCLUSIONS}

We have presented a novel yet practical endless SOP controlling system for use in coherent fiber-optic detection systems which uses three polarization controlling devices constructed with nematic liquid crystals. We have illustrated the importance of polarization matching in heterodyne detection and the fundemental operation of liquid crystal retarders. Finally, we provided an in-depth description of the system control and demonstrated it experimentally. 


\section{REFERENCES}

[1] B. Basch, R. Kearns, G. Joyce, S. Stone, W. Chen, "Progress toward coherent fiber transmission," Proceedings of SPIE, Coherent Technology in Fiber Optic Systems, vol. 568, August 19, 1985.

[2] T. Okoshi, "Heterodyne and coherent optical fiber communications: recent progress", IEEE Transactions on MicrowaveTheory and Techniques, vol. MTT-30, no. 8, p. 1138, August 1982.

[3] Y. Yamamoto, T. Kimura, "Coherent optical fiber transmission systems", Journal of Quantum Electonics, vol. QE-17, no. 6, p. 919, June 1981.

[4] N. G. Walker, G. R. Walker, J. Davidson, D. C. Cunningham, A. R., Beaumont, R. C. Booth, "Lithium niobate waveguide polarisation converter", Electronics Letters, vol. 24, no. 2, p. 103, January 21, 1988.

[5] E. E. Basch, T. G. Brown, "Introduction to coherent optical fiber transmission", IEEE Communications Magazine, vol. 23, no. 5, p.23, May 1985.

[6] R. A. Harmon, "Polarization stability in long lengths of monomode fibre," Electronics Letters, vol. 18, no. 24, p. 1058,November 25, 1982.

[7] H. Kobrinski, "Applications of coherent optical communication in the network environment," Proceedings of SPIE, Coherent Technology in Fiber Optic Systems, vol. 568, August 19, 1985. 
[8] N. G. Walker, G. R. Walker, "Endless polarisation control using four fibre squeezers," Electronics Letters, vol. 22, no. 6, 1987.

[9] T. Matsumoto, R. Nagase, and H. Kano, "Proposal for a fiber-optic endlessly rotatable fractional wave device and its applications to lightwave technologies," Elecronics and Comunications in Japan, Part 2, vol. 71, no. 6, pp.36-47, 1988.

[10] R. Noe, "Endless polarisation control in coherent optical communicatons", Electronic Letters, vol. 22, no. 15, July 17, 1986.

[11] M. Johnson, "In-line fiber-optical polarization transformer", Applied Optics, vol. 18, no. 9, p.1288, May 1, 1979.

[12] R. Ulrich, "Polarization stabilization on single-mode fiber", Appl. Phys. Lett., vol. 35, no. 11, p. 840, December 1, 1979.

[13] T. Matsumoto, H. Kano,"Endlessly rotatable fractional-wave devices for single-mode-fibre optics", Electronics Letters, vol. 22, no. 2, p. 78, January $16,1986$.

[14] R. Ulrich and M. Johnson, "Single-mode fiber-optical polarization rotator", Applied Optics, vol. 18, no. 11, p.1857, June 1, 1979.

[15] Rod C. Alferness, "Electrooptic guided-wave device for general polarization transformations", Journal of Quantum Electronics, vol. QE-17, no. 6, p. 965, 1981.

[16] R. Noe, H. Heidrich, D. Hoffman, "Automatic endless polarization control with intedgrated-optical $\mathrm{Ti}: \mathrm{LiNbO}_{3}$ polarization transformers", Optics Letters, vol. 13, no. 6, p. 527, June 1988.

[17] J. Slaz, "Coherent lightwave communications", AT\&T Technical Journal, vol. 64, no. 10, p. 2153, December 1985. 
[18] N. G. Walker, G. R. Walker, J. Davidson, "Endless polarisation control using an integrated optic lithium niobate device", Electronics Letters, vol. 24, no. 5, p. 266, March 3, 1988.

[19] T. Okoshi, " Polarization-state control schemes for heterodyne or homodyne optical fiber communications," Journal of Lightwave Technology, volume LT-3, number 6, December 1985

[20] T. Imai, K. Nosu, H. Yamaguchi, "Optical polarisation control utilising an optical heterodyne detection scheme," Electronics Letters, vol. 21, no.2, p. 52, January 17, 1985.

[21] S. Rumbaugh, W. Casperson, M. Jones, and P. Bos, "Endless state-of-polarization control for coherent optical communications using liquid crystals," Proceedings of the Oregon Academy of Science, Portland, Oregon, Feburary 5 - 6, 1988.

[22] S. Rumbaugh, M. Jones, L. Casperson, "Polarization control for coherent fiber-optic communications using nematic liquid crystals," to be published in the Journal of Lightwave Technology.

[23] D. Armitage, " Liquid crystal voltage controlled retardation display,"Applied Optics, vol. 19, no.13, p. 2235, July 1, 1980.

[24] A. Yariv, Quantum electronics, John Wiley \& Sons, p. 345, New York, 1975

[25] R. Jones, "A new calculus for the treatment of optical systems I. description and discussion of the calculus," Journal of the Optical Society of America, vol. 31, p. 488, July 1941.

[26] R. Jones, "A new calculus for the treatment of optical systems II. proof of three general equivalence theorems," Journal of the Optical Society of America, vol. 31, p. 493, July 1941. 
[27] R. Jones, "A new calculus for the treatment of optical systems III. The sohnke theory of optical acitvity," Journal of the Optical Society of America, vol. 31, p. 488, July 1941.

[28] W. Shurcliff, Polarized light: Production and Use, Harvard University Press, pp. 109-123, Cambridge Massachusetts, 1966.

[29] Ibid, pp. 15-19. 


\section{APPENDIX}

\section{POLARIZED LIGHT}

\section{INTRODUCTION}

The polarization of a monochomatic beam may be determined by the magnitude and phase of the field in two orthogonal directions transverse to the direction of propagation. Thus, polarization may be altered passing the beam through an optically anisotropic material which introduces a phase delay in one field direction with respect the other. Jones Calculus is a mathmatical construct for determining the effects of anisotropic or dichroic materials on polarized light and Poincare's Sphere is a graphical method for the same means. These methods are used extensively in this thesis and are presented below.

\section{JONES CALCULUS}

The effects of a system of polarization controlling devices on polarized light can be calculated with Jones Calculus [25], [26], [27], [28]. In Jones Calculus, the electric field polarization is represented by a vector in the form,

$$
\overrightarrow{\mathbf{E}}=\left[\begin{array}{l}
A_{x} e^{i \varepsilon x} \\
A_{y} e^{i \varepsilon y}
\end{array}\right]
$$


where $A_{x}$ and $A_{y}$ are the amplitudes of the field in the $x$ and $y$ directions, and their phase difference is $\varepsilon_{\mathrm{y}}-\varepsilon_{\mathrm{x}}$. In general, the amplitude of the total field is normalized to unity.

A birefringent device may be used to change polarization and is represented by a 2 by 2 matrix. The resultant polarization is found by multiplying input polarization vector by device matrix. The Jones matrix for a linearly birefringent device, such as the liquid crystal retarders used here, which is aligned to the principal axes is,

$$
\left[\begin{array}{cc}
\mathrm{e}^{\mathrm{i} \rho / 2} & 0 \\
0 & \mathrm{e}^{-\mathrm{i} \rho / 2}
\end{array}\right]
$$

where $\rho$ is the retardance. The matrix for the same devices tilted 45 degrees is,

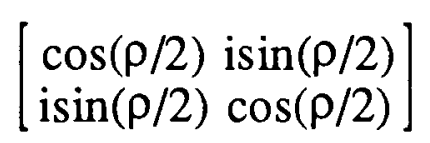

\section{POINCARE SPHERE}

The Poincare sphere (see Figure 12) is a three dimensional model useful in describing the effect of optical phase retarders on polarized light. [29] Every point on the sphere represents a unique polarization state. Linear states are mapped on to the equator with right-handed and lefthanded elliptic states mapped to the hemispheres above and below respectively. Ellipticity of the states increases in the direction of the poles which represent the circular states. lines of constant ellipticity defined by 
lines of lattitude and lines of constant tilt are defined by longitudinal lines. Orthogonal polarizations are dimeterically oposite of each other. The $\mathbf{H}$, V, RC, and LC indicate the horizontal linear, vertical linear, right-handed circular and left-handed circular states.

Polarization conversions by birefringent devices are represented as coordinate transformation on Poincare's sphere. The effect of a retarder on an arbitrary input polarization state is determined by a rotation on the surface of the sphere about an axis defined by the two orthogonal eigenaxes of the retarder from the point corresponding to the input polarization to that of the output polarization. The angle of rotation is equal to the optical phase retardation. The retarders have linear eigen-axes so the axis of rotation intercepts the equator at a point corresponding the the tilt of the retarder's eigen axes.

An example of a quarter-wave retarder tilted 45 degrees to $\mathrm{H}$ is illustrated in Figure 13. The longitude corresponding to 45 degree tilt is located by rotating 90 degrees around the equator in a counter clock-wise direction as viewed by the $\mathbf{R}$ state. This point is indicated with an FA and inicating the fast-axis. The SL is diameterically opposite and inicates the location of the slow axis. If the input beam is linearly polarized and vertical, the output polarization is found by rotating 90 degrees about FASL. Thus, the output beam is right-handed circularly polarized. 


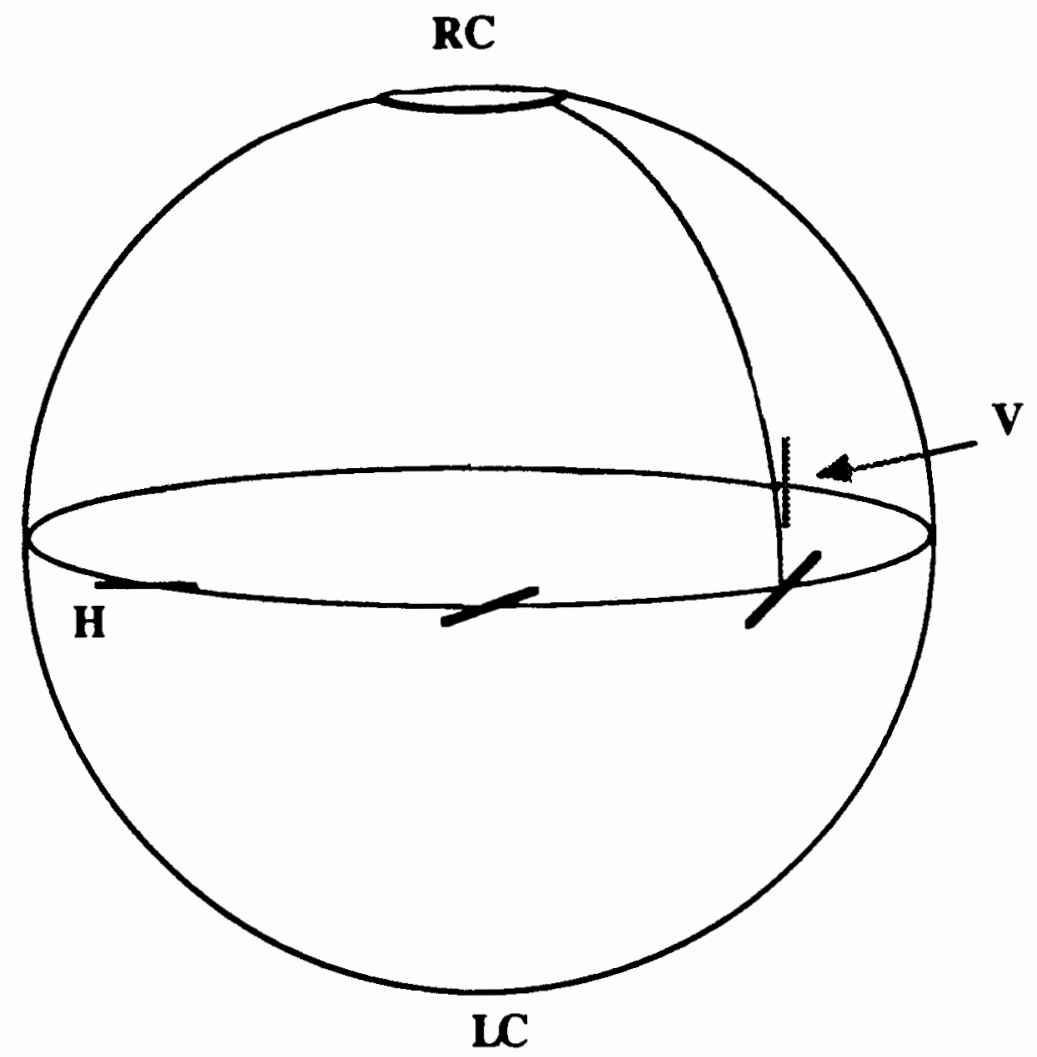

Figure 12. The poincare sphere.

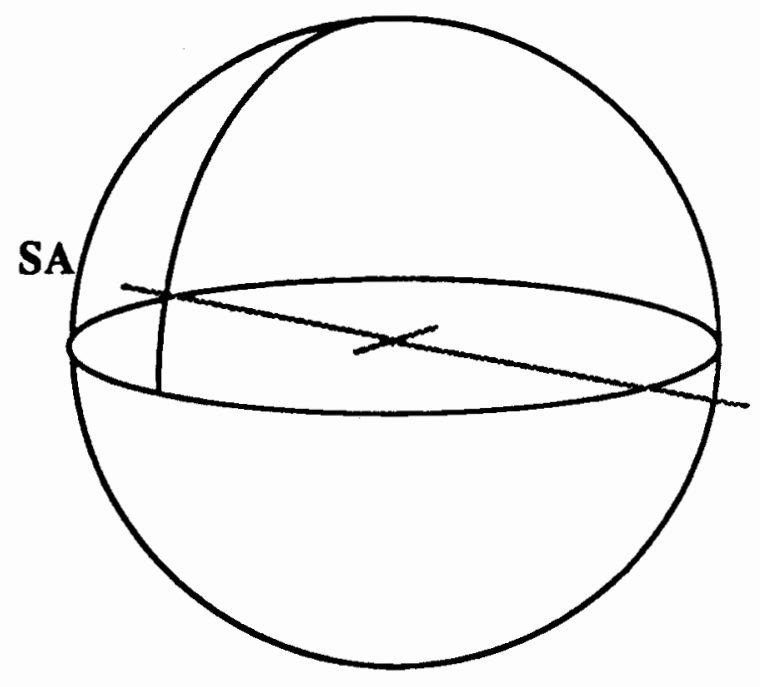

Figure 13. An example using the Poincare sphere. 